\title{
RAISING IN WATERSHED LATTICES
}

\section{Jean Cousty}

\author{
INRIA Sophia-Antipolis - ASCLEPIOS team, \\ France,jean.cousty@inria.fr
}

Laurent Najman and Jean Serra

\author{
Université Paris-Est, LABINFO-IGM,
} UMR CNRS 8049, A2SI-ESIEE, France

\begin{abstract}
The watershed segmentation is a popular tool in image processing. Starting from an initial map, the border thinning transformation produces a map whose minima constitute the catchment basins of the watershed of the initial map. An interesting feature of the transformed map (called border kernel) is to convey not only the watershed partition but also numeric information relative to the initial map. In this paper, we provide the space of all border kernels with a semi lattice and propose morphological operations (relative to this lattice) which allow for merging border kernels and building hierarchies of watersheds based in particular on connected filters.
\end{abstract}

Index Terms - Watershed, border kernels, lattice, hierarchies, data merging, mathematical morphology

\section{INTRODUCTION}

The watershed segmentation is a popular tool in image processing [1-3]. It is often combined with connected filters $[4,5]$ which simplify the considered function (also called map in this paper), and lead to watershed partitions with larger classes. However, it is not only a matter of "partition growing": a connected filter by flat zones increases the partition by flat zones of the considered function, but it does not necessarily increase its watershed partition. Indeed, the crest lines (thus, the watershed) of the map obtained after the filtering step may be "shifted" compared to the ones of the original map (see Fig. 1, where $F$ is the initial map, $F_{3}$ a watershed of $F, F_{4}$ a filtering of $F_{3}$, and $F_{5}$ a watershed of $F_{4}$ ).

On the other hand, there is an ambiguity in the very definition of a watershed. In some cases, we want to effectively build the crest lines (divide lines). In some other cases, we are interested in the partition of the space into its catchment basins and, in this case, we do not know on which side lies the crest lines.

Recent works by J. Cousty et al. [6,7] allow us to make precise these notions at least in the discrete cases of finite edge-weighted graphs. Starting from the space $E$ made of the edges of a graph rather than of its vertices, an original idea consists of introducing the border thinnings on the edge maps. Let us briefly recall these notions.
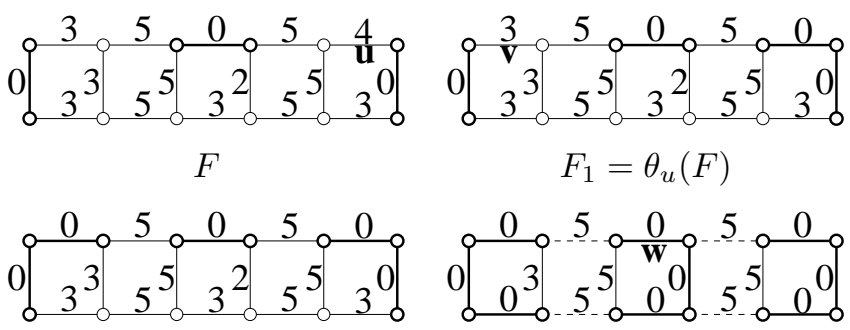

$$
F_{2}=\theta_{v}\left(F_{1}\right)
$$

$$
F_{3}=\check{\theta}(F)
$$
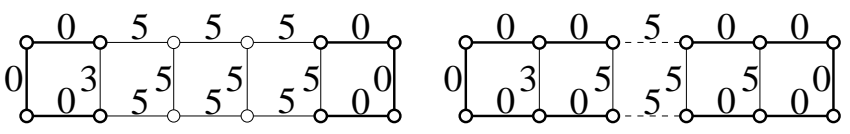

$$
F_{4}=\check{\eta}_{w}\left(F_{3}\right)
$$

$F_{5}$

Fig. 1. Watershed and connected filtering in edge-weighted graphs. The map $F_{3}$ is a border kernel of $F$ and the dashed edges a watershed of $F$. The map $F_{4}$ is obtained from $F_{3}$ thanks to a connected filter (a flooding see Sec. 4). The map $F_{5}$ is a border kernel of $F_{4}$ and the dashed edges is a watershed of $F_{4}$. Minima are depicted in bold.

Hereafter, the workspace is a finite family of edges $E$ (i.e., $E$ is a set made of pairs of points), whose extremities define the vertex space $E^{*}$. Any element $X \subseteq E$ induces a family of vertices $X^{*}$ in $E^{*}$. The class made of the maps $F: E \rightarrow \mathbb{K}$ (where $\mathbb{K}$ is any finite subset of $\mathbb{Z}$ ) is denoted by $\mathcal{F}$. A map $F \in \mathcal{F}$ weights the edges of $E$.

Given a map $F \in \mathcal{F}$, to define the border thinnings, it is convenient to also consider $F^{*}: E^{\star} \rightarrow \mathbb{K}$ which maps each element $x$ in $E^{*}$ to the minimal value of an edge in $E$ that contains $x$, i.e., $F^{*}(x)=\min \{F(u) \mid u \in E$ and $x \in u\}$. Let $u=\{x, y\} \in E$, we say that

- $u$ is a separating edge (for $F$ ) if $F(u)>\max \left(F^{*}(x)\right.$, $\left.F^{*}(y)\right)$

- $u$ is a border edge (for $F$ ) if $F(u)=\max \left(F^{*}(x)\right.$, $\left.F^{*}(y)\right)$ and $F(u)>\min \left(F^{*}(x), F^{*}(y)\right)$; and that

- $u$ is an inner edge (for $F$ ) if $F^{*}(x)=F^{*}(y)=F(u)$.

The border thinnings are the idempotent applications $\check{\theta}$ acting on $\mathcal{F}$ and generated by compositions of the elementary 
operator $\theta_{u}$, with $u \in E$ :

$$
\begin{aligned}
\left(\theta_{u} F\right)(u) & =\min _{x \in u}\left\{F^{*}(x)\right\} \text { if } u \text { is a border edge for } F, \\
\left(\theta_{u} F\right)(u) & =F(u) \text { if } u \text { is not a border edge for } F, \\
\left(\theta_{u} F\right)(v) & =F(v) \text { if } v \neq u .
\end{aligned}
$$

The map $\check{\theta}(F)$ obtained by a border thinning is called a border kernel (of $F$ ). These notions are illustrated in Fig 1 by the maps $F, F_{1}, F_{2}$ and $F_{3}$. Any edge of a border kernel is either an inner edge (in this case, it belongs to a minimum) or a separating edge (in this case it is not in a minimum but its extremities are both in a minimum). Therefore, any border kernel induces a (connected) partition of $E^{*}$. Each class of the partition is a set of vertices induced by all edges in a single minimum. It satisfies the strong following properties.

Theorem 1 ( $[6,7]){ }^{1}$ If $H$ is a border kernel of $F$ then:

$1 /$ the set $S$ of all edges in $E$ whose extremities are in two distinct minima of $H$ is a watershed of $F$ and furthermore for any $u \in S, H(u)=F(u)$; and

$2 /$ the union of all minima of $H$ is a minimum spanning forest for $F$ relative to the minima of $F$.

This theorem invites us to study the structure of the set of all border kernels. Indeed, building a hierarchy of watersheds means that we are able to define an order relation over this set. Could we go further and build a lattice? It would then allows us to construct pyramids of watersheds and to combine watersheds stemming from several sources. How could we furthermore describe basic operations (e.g., dilations, closings) on this lattice and study their properties? We begin in this paper to investigate such structures and operators.

\section{LATTICE OF BORDER KERNELS}

Even if two distinct series $\left\{\theta_{u_{p}}\right\}$ can lead to two distinct limit products $\check{\theta}_{1}$ and $\check{\theta}_{2}$, we always have $\check{\theta}_{2} \check{\theta}_{1}(F)=\check{\theta}_{1}(F)$, since, by definition, there is no border edge for $\theta_{1}(F)$. Similarly, $\check{\theta}_{1} \check{\theta}_{2}(F)=\check{\theta}_{2}(F)$. It means that the invariance domain of the thinnings $\check{\theta}_{i}$ is the same for all thinnings $\check{\theta}_{i}$. It is the set of all border kernels, or said differently the maps in $\mathcal{F}$ for which there is no border edge. For the sake of simplicity, in this paper, we will only consider the border kernels whose minima are all of altitude 0 (the minimal value of $\mathbb{K}$ ) and we will denote this set by $\mathcal{A}$.

It is convenient to provide the space $\mathcal{A}$ with the order by minima, or min order. Let $F$ and $G \in \mathcal{A}$. Denote by $\mathcal{M}(F)$ and $\mathcal{M}(G)$ the sets of all edges lying in the minima of $F$ and $G$ respectively, and denote by $\mathcal{S}(F)$ and $\mathcal{S}(G)$ the sets of their separating edges $(\mathcal{M}(F) \cup \mathcal{S}(F)=E)$. Map $F$ is said smaller than map $G$ for the min order, written $F \preceq G$, if:

1/ $\mathcal{M}(F) \subseteq \mathcal{M}(G)$, or equivalently $\mathcal{S}(F) \supseteq \mathcal{S}(G)$

2/ $F(u) \leq G(u)$ for any edge $u \in \mathcal{S}(G)$.

\footnotetext{
${ }^{1}$ The reader can refer to [6] for the precise definitions of a watershed (cut) and of a relative minimum spanning forest, as considered in Theorem 1.
}

We call hierarchy (of border kernels) any sequence $\left(F_{0}, \ldots, F_{n}\right)$ of maps in $\mathcal{A}$ such that $F_{0} \preceq \cdots \preceq F_{n}$.

For instance, $F_{3}$ (Fig. 1) is smaller than $F_{7}$ (Fig. 2) but it is not smaller than $F_{5}$ (Fig. 1) since $\mathcal{S}\left(F_{3}\right) \nsupseteq \mathcal{S}\left(F_{5}\right)$.

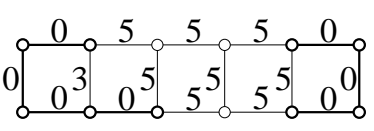

$F_{6}$

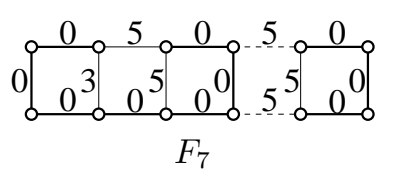

Fig. 2. Map $F_{6}$ is obtained from $F_{4}$ by a first step of thinning constrained by the kernel $F_{3}$ and $F_{7}$ is a border kernel of $F_{4}$ (obtained by a second step of thinning) constrained by $F_{3}$.

Theorem 2 (Border kernel sup-semi lattice) The min order generates on $\mathcal{A}$ a sup-semi lattice, denoted $\curlyvee$, whose greatest element is the constant border kernel $F_{\max }$ for which the weight of each edge equals to 0 (the minimal value of $\mathbb{K}$ ). For any two elements $F_{1}$ and $F_{2}$ in $\mathcal{A}$ there exists a smallest upper-bound $F=F_{1} \curlyvee F_{2}$, or supremum defined by:

$$
\begin{aligned}
& F(u)=\max \left\{F_{1}(u), F_{2}(u)\right\} \text { if } u \in \mathcal{S}\left(F_{1}\right) \cap \mathcal{S}\left(F_{2}\right) \\
& F(u)=0 \text { otherwise. }
\end{aligned}
$$

Furthermore, the partition of $E^{*}$ induced by $F_{1} \curlyvee F_{2}$ is equal to the partition $D=D_{1} \vee D_{2}$, where $D_{1}$ (resp. $D_{2}$ ) is the partition induced by $F_{1}$ (resp. $F_{2}$ ) and where $\vee$ denotes the supremum of partitions [8].

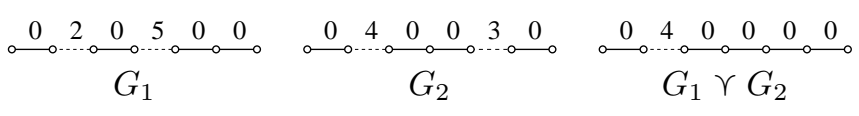

Fig. 3. Illustration of the supremum $\curlyvee$ for the min order.

This definition of a supremum in $\mathcal{A}$ is illustrated Fig. 3.

On the other hand, two kernels $F_{1}$ and $F_{2}$ in $\mathcal{A}$ do not necessarily admit an infimum since the intersection of the classes induced by $F_{1}$ and $F_{2}$ can lead to classes reduced to a single vertex and there is no set of edges that induces such a class. However, if we set, by hypothesis, a smallest border kernel $F_{0}$ (the zero element of a hierarchy), then the family of all border kernels greater than $F_{0}$ is a complete lattice (for the min order). Indeed $\curlyvee$ is still the supremum for this family and, since $F_{1} \succcurlyeq F_{0}$, and $F_{2} \succcurlyeq F_{0}$, the set of the border kernels less than both $F_{1}$ and $F_{2}$ is not empty and this family admits a greater elements $F_{1} \curlywedge F_{2}$.

Corollary 3 Let $F_{0} \in \mathcal{A}$. The family $\mathcal{A}_{0}$ of the elements in $\mathcal{A}$ greater than $F_{0}$ is a complete lattice whose supremum is the one of $\mathcal{A}$ and whose infimum is defined by:

$$
\begin{aligned}
F(u)= & \min \left\{F_{1}(u), F_{2}(u)\right\} \text { if } u \in \mathcal{S}\left(F_{1}\right) \cap \mathcal{S}\left(F_{2}\right) \text { or } \\
& u \in \mathcal{M}\left(F_{1}\right) \cap \mathcal{M}\left(F_{2}\right) \\
F(u)= & \max \left\{F_{1}(u), F_{2}(u)\right\} \text { otherwise. }
\end{aligned}
$$




\section{RAISINGS}

Which operations can we build for acting on the sup-semi lattice of border-kernels $\mathcal{A}$ ? The existence of a supremum orients us towards dilations, and, since this supremum $\curlyvee$ extends the minima, we take as the basic operation the elementary raising $\rho_{u}$ defined below.

Fix an edge $u$ that parametrizes the operation $\rho_{u}$ and define, for any $F \in \mathcal{A}$, the elementary raising by edges by:

$$
\begin{aligned}
& \rho_{u} F(u)=0 \text { if } u \text { is adjacent to two distinct minima of } F \\
& \rho_{u} F(u)=F(u) \text { if } u \text { is adjacent to one minimum of } F \\
& \rho_{u} F(v)=F(v) \text { if } v \neq u
\end{aligned}
$$

Theorem 4 Let $u \in E$. The raising $\rho_{u}$ acts on $\mathcal{A}$ and is both a closing and a dilation with respect to the min order.

In other words, $\rho_{u}$ satisfies the five following properties:

i) $\rho_{u}(F) \in \mathcal{A}$;

ii) $\rho_{u}\left(F_{1} \curlyvee F_{2}\right)=\rho_{u}\left(F_{1}\right) \curlyvee \rho_{u}\left(F_{2}\right)$;

iii) $F_{1} \preceq F_{2}$ implies $\rho_{u}\left(F_{1}\right) \preceq \rho_{u}\left(F_{2}\right)$;

iv) $F \preceq \rho_{u}(F)$; and

v) $\rho_{u} \rho_{u}(F)=\rho_{u}(F)$.

Furthermore, any product $\check{\rho}_{k}$ obtained by successive compositions of a series $\left\{\rho_{u_{j}} \mid j \in[1, k]\right\}$ (i.e., $\check{\rho}_{k}=\rho_{u_{k}} \ldots \rho_{u_{1}}$ ) are still dilations and closings. Thus, by iv), the successive terms $\left\{\check{\rho}_{j}\right\}$ generate a hierarchy of border kernels.

\section{FLOODINGS}

The flooding operation $\check{\eta}$, which we consider in this section, is a connected operator and it constitutes an interesting way for obtaining a raising. F. Meyer and L. Najman [9] define it as any extensive operator acting on $\mathcal{F}$ such that:

$$
\begin{aligned}
{[(\check{\eta} F)(u)} & >\max [(\check{\eta} F)(v), v \text { adjacent to } u] \\
\Rightarrow \quad(\check{\eta} F)(u) & =F(u) .
\end{aligned}
$$

The previous property is rather a characteristic property than a direct definition. In fact, we can show that any flooding $\check{\eta}$ can be obtained as a composition product of elementary floodings $\eta_{u}$ associated to any edge $u \in E$ and defined by:

$$
\begin{aligned}
\left(\eta_{u} F\right)(v)= & F(v)+1 \text { if } u \text { and } v \text { belong to a same } \\
& \text { minimum } \\
\left(\eta_{u} F\right)(v)= & F(v) \text { otherwise. }
\end{aligned}
$$

The floodings (see for instance $F_{4}=\check{\eta}_{w}\left(F_{3}\right)$ in Fig. 1), that often give nice results, as connected filters in $\mathcal{F}$, do not allow for producing a hierarchy of watersheds, even if we apply them to a map in $F \in \mathcal{A}$ (see the counter-example $F_{5}$ in Figure 1). To obtain a hierarchy from floodings, we have to complete them with a class of constrained border thinnings (more details will be provided in an extended version [10]).
The maps $F_{3}, F_{4}$ (Fig. 1), $F_{6}$ and $F_{7}$ (Fig. 2) illustrates the four steps of the composition of a flooding $\check{\eta}_{w}$ with a constrained border thinning $\check{\zeta}$, whose product is indeed a raising and thus allows for producing a hierarchy of border kernels.

Hence, to produce a hierarchy of border kernels based on floodings and constrained border thinnings, one only needs a sequence of edges to parametrize successive floodings. To this end, given an initial border kernel, one may select one edge per minimum and order these edges thanks to attributes relative to the minima (area, dynamics, ..., $[5,11,12])$. Then, we can construct a hierarchy of border kernels, hence a hierarchy of watersheds. These watersheds can be stacked to build a new map so that each edge is weighted by the number of watersheds it belongs to. Such a map is called a saliency map [13]. Note that any saliency map is a border kernel.

The saliency maps $S_{1}$ and $S_{2}$ obtained from Fig. 4 thanks to dynamics and surface attributes are depicted in Fig. 5. The saliency $S_{1}$ correctly discriminates the significant contours but it also strongly delineates many small highly-contrasted regions which correspond to noise. On the other hand, $S_{2}$ does not discriminates these noisy regions, but it divides some large homogeneous zones of the image into several parts. How could we combine the advantages of these two hierarchies? The framework settled in this paper precisely provides an answer: the infimum of $S_{1}$ and $S_{2}\left(S_{1} \curlywedge S_{2}\right)$ is depicted in Fig. 5. In the second row of Fig. 5, we also show the segmentations into 100 regions obtained from $S_{1}$, $S_{2}$ and $S_{1} \curlywedge S_{2}$.

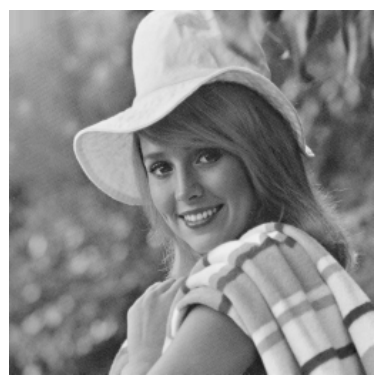

Fig. 4. A grayscale image.

\section{CONCLUSION}

In this paper, we associated a semi lattice structure to the family of border kernels. Based on this, we showed how to merge elements of this family and proposed a generic morphological operation to build hierarchies of border kernels. We outlined how to obtain interesting hierarchies based on the flooding connected-operator and how to merge these hierarchies.

The floodings only deal with scalar functions (so that we can define minima). However, raisings are adapted to data fusion, and can handle simultaneously several minima since they are dilations. Future works will focus on this last point. 


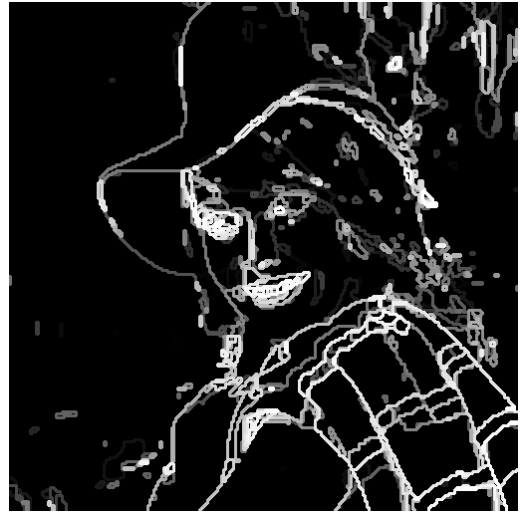

$S_{1}$

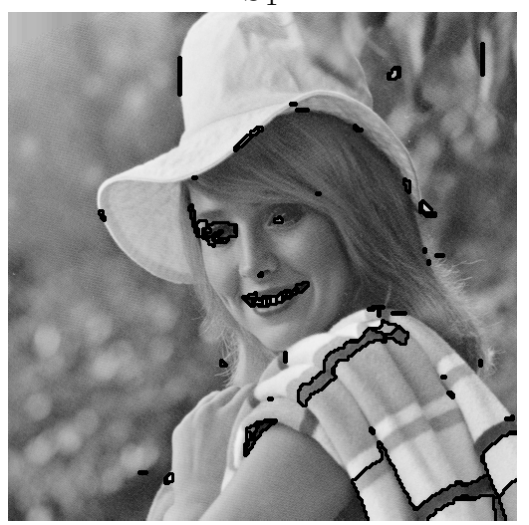

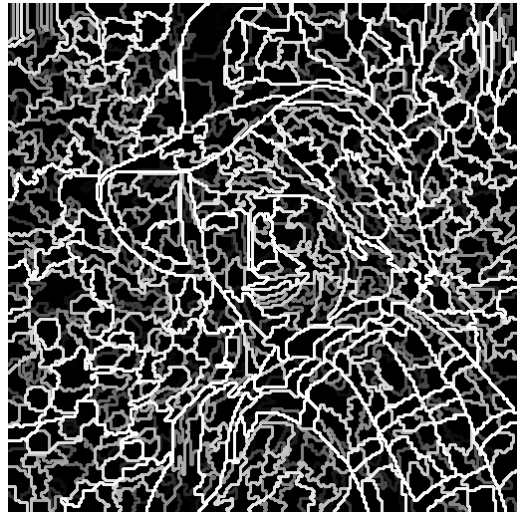

$S_{2}$

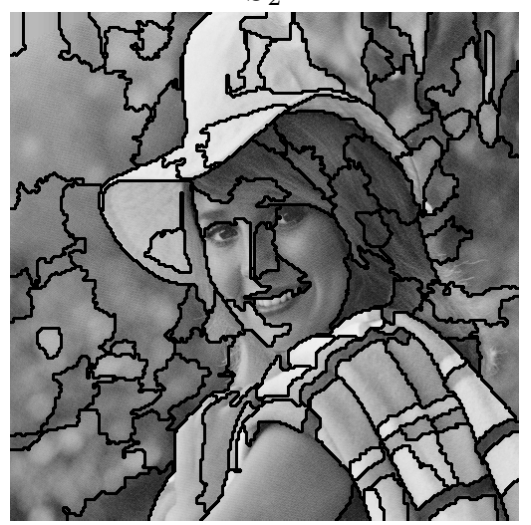

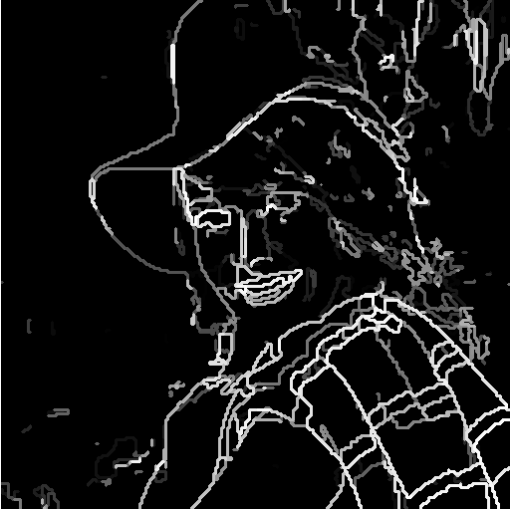

$S_{1} \curlywedge S_{2}$

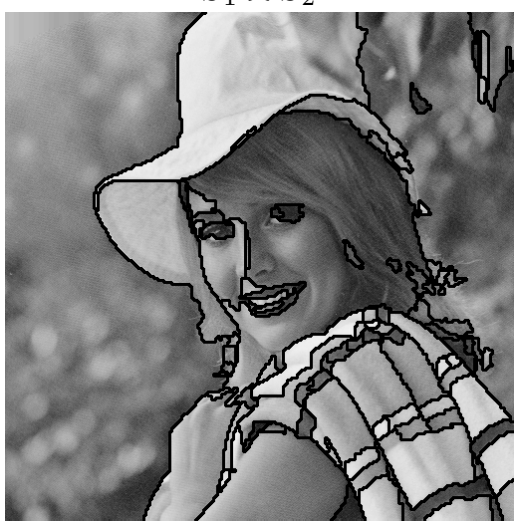

Fig. 5. Saliency maps obtained from Fig. 4 (first row) and associated segmentations into 100 regions (second row) [see text].

\section{REFERENCES}

[1] S. Beucher and F. Meyer, "Morphological segmentation," Journal of Visual Communication and Image Representation, vol. 1, pp. 21-46, 1990.

[2] L. Vincent and P. Soille, "Watersheds in digital spaces: An efficient algorithm based on immersion simulations," IEEE PAMI., vol. 13, no. 6, pp. 583-598, June 1991.

[3] G. Bertrand, "On topological watersheds," JMIV, vol. 22, pp. 217-230, 2005.

[4] P. Salembier and J. Serra, "Flat zones filtering, connected operators and filters by reconstruction," IEEE TIP, vol. 4, no. 8, pp. 1153-1160, 1995.

[5] E. Breen and R. Jones, "Attribute openings, thinnings and granulometries," CVIU, vol. 64, pp. 377-389, 1996.

[6] J. Cousty, G. Bertrand, L. Najman, and M. Couprie, "Watersheds, minimum spanning forest and the drop of water principle," 2007, Technical Report IGM2007-01, http://igm.univmlv.fr/LabInfo/rapportsInternes/2007/01.pdf, also submitted for publication.
[7] J. Cousty, G. Bertrand, L. Najman, and M. Couprie, "On watershed cuts and thinnings," in procs. DGCI, 2008, vol. LNCS 4992, pp. 434-445.

[8] J. Serra, "A lattice approach to image segmentation," JMIV, vol. 24, pp. 83-130, 2006.

[9] F. Meyer and L. Najman, Morphologie Mathématique: approches déterministes, chapter Segmentation, arbre de poids minimum et hiérarchies, Hermes Sciences, 2008, to appear.

[10] J. Cousty, L. Najman, and J. Serra, "Raising and watershed lattices," In preparation.

[11] C. Vachier, Extraction de caractristiques, segmentation d'images et morphologie mathématique, Ph.D. thesis, Ecole des Mines, Paris, 1995.

[12] G. Bertrand, "On the dynamics," Image Vision Comput., vol. 25, no. 4, pp. 447-454, 2007.

[13] L. Najman and M. Schmitt, "Geodesic saliency of watershed contours and hierarchical segmentation," IEEE PAMI., vol. 18, no. 12, pp. 1163-1173, December 1996. 